\title{
Risk of Thromboembolic Disease and Hemorrhage in Patients with Multiform Glioblastoma
}

\author{
Iris Violeta de la Rocha Vedia, Jose Portillo Sanchez \\ Internal Medicine, General University Hospital of Ciudad Real, Ciudad Real, Spain
}

\section{Email address:}

Iris_delarocha@yahoo.es (I. V. de la R. Vedia)

\section{To cite this article:}

Iris Violeta de la Rocha Vedia, Jose Portillo Sanchez. Risk of Thromboembolic Disease and Hemorrhage in Patients with Multiform Glioblastoma. American Journal of Internal Medicine. Vol. 9, No. 3, 2021, pp. 153-159. doi: 10.11648/j.ajim.20210903.18

Received: May 8, 2021; Accepted: May 31, 2021; Published: June 9, 2021

\begin{abstract}
Background: Glioblastoma multiforme (GBM) is the most frequent malignant brain tumor, with an aggressive course and a short life expectancy despite standard treatment. The possibility of the development of thrombotic events (VTE) with this type of cancer is frequent. Objective: To determine the risk of presenting VTE and haemorrhagic events in patients affected by GBM. Methods: Observational retrospective study of patients with GBM diagnosis at the General University Hospital of Ciudad Real between 2012 and 2015. The demographic characteristics of patients were studied, predictive models were compared, and a survival analysis was performed. Results: 13 (16.9\%) out of 77 patients developed symptomatic VTE. The quality of life according to the performance status ECOG scale at the moment of diagnosis was 1 in 42 (15.38\%) patients, and at the time of VTE, $5(41.7 \%)$ had a value of 2, and $4(33.3 .3 \%)$ registered 3. In the group that developed VTE according to the predictive model of risk for thrombosis in Khorana $5(38.5 \%)$ had low risk and $8(61.5 \%)$ intermediate; on the ASCO 2013 modified scale $5(38.5 \%)$ had an Intermediate risk and $8(61.5 \%)$ high. With a median, 1 year follow-up, $64(84.2 \%)$ patients died, with an average time after diagnosis of 279.09 days (216.6-341.6) (SE 31,8). $2(2.6 \%)$ of the patients presented a greater haemorrhagic event and 7 (7.9\%) cerebral haemorrhage, of which 4 (44.4\%) had prophylactic Low molecular weight heparins (LMWHs). In the survival analysis, patients who received prophylactic treatment with LMWHs had a higher survival rate with an average of 298.5 days compared to 239.3 of those who did not ( $>>0.05)$. There were no significant variables in the multivariate analysis for thrombotic or haemorrhagic events. Conclusion: The demographic and clinical characteristics of our patients were similar to those reported in other publications. The predictive scale of Khorana was not validated in our study, in contrast, the modified ASCO 2013 scale was closer to our results. The creation of a precise predictive model would help to delineate the benefit of prophylactic anticoagulation in high-risk patients. Long-term prophylaxis with LMWHs has demonstrated a reduction of thrombotic events without significantly increasing fatal haemorrhagic episodes, also demonstrating greater long-term survival, independent of thrombotic events. Randomized prospective studies are needed to demonstrate its benefits.
\end{abstract}

Keywords: Glioblastoma Multiforme, Thrombosis, Haemorrhagic, Low Molecular Weight Heparins

\section{Introduction}

The medical scientific community is concerned about the limited vital expectations of patients with brain tumors; which also have, as a frequent complication, thrombotic events, particularly those affected by GBM, which are unacceptably high. This type of tumor, being the most frequent of cerebral primaries, presents the drama of scarce survival, which stands at 14 months on average despite therapeutic efforts. The prognosis of these tumors has not varied in recent decades regardless of surgery, chemo and radiation therapy or the introduction of new drugs, which makes new approaches and multidisciplinary cooperative work, necessary as a useful strategy [1].

In patients with cancer, thromboembolic complications are included among the main causes of death. In the Multinational Register RIETE (Registro Informatizado de Enfermedad Tromboembólica) it was found that the threemonth mortality rate after a thrombosis was significantly higher in cancer patients $(26.4 \%$ vs $4.1 \%)$ [2]. It has been shown that treatment with anticoagulants, especially LMWHs, improves survival in oncological patients, not only 
due to the decrease in thromboembolic events, but also as a result of the possibility of its antineoplastic activity in different types of tumors [3-5].

The characteristic necrosis that can be viewed in the GBM, is suspected to be due to regions of hypoxia, therefore, it is possible that the combination of low levels of oxygen and the intrinsic biology of the tumors, are responsible for necrosis in pseudoempalized. In fact, Brat et al, propose that pseudoempalties are generated by a migratory activation of hypoxia in the cell population, whose theory explains that, after thrombosis of one of the blood vessels due to the excessive growth of tumor cells and the secretion of thrombotic factors, the local oxygen decreases promoting a massive migration of hypoxic glioma cells towards better oxygenated areas. In the process, hypoxic glioma cells are infiltrated by normoxic glioma cells that are closer to a functional vessel, creating a transient region of hypercellularity (one pseudoempalized). As pseudomepalized is enlarged around the thrombosed vessel, perivascular necrosis becomes more prominent [6].

Given that on the Korana scale [7], designed for patients with breast cancer, is not an adequate model for the stratification of thrombotic risk in patients with brain tumors, the only validated scale which may be more useful is the one modified by the ASCO (American Society of Clinical Oncology) 2013, which includes this type of pathology as a high risk [8].

In the latest studies of thromboprophylaxis survival, they have focused on pancreas, lung, breast and gynecological cancers, without including data of brain tumors, as is the case with the GBM, which had been considered low risk but, paradoxically, considered by literature as high thrombotic risk [9].

\section{Material and Methods}

This study was approved by the Ethics Committee of Gerencia de Atención Integrada de Ciudad Real. We carried out a retrospective observational study of GBM diagnosis (Glioma grade IV of the World Health Organization (WHO)) at the General University Hospital of Ciudad Real, between 2012 and 2015. Its clinical characteristics were analyzed (hypertension, diabetes, chronic obstructive pulmonary disease, dyslipemia), functional status (KPS score) at the start of the chemotherapic treatment, the type of surgery, concomitance or not, with temozolomide $\left(75 \mathrm{mg} / \mathrm{m}^{2} /\right.$ day $\mathrm{x}$ six weeks) and radiotherapy prescribed to 60 gy 30 daily fractions divided during the same period, equally in patients who developed thrombotic events or not. Different predictive scales were evaluated for thrombosis (korana, ASCO modified), as a hemorrhage (HASBLED).

To collect the information, a manual registration form was created in a database designed in the PASW Statistic program, version 18 .

\section{Results}

Between 2012 and 2015, 77 patients were diagnosed with GBM, the data was obtained from the brain tumor committee registry.

Table 1. Characteristics of patients with and without thrombotic events.

\begin{tabular}{lllll}
\hline Results & & Patients with VTE & Patients without VTE & All patients \\
\hline Total & & 13 & 64 & 77 \\
Gender & Male & $6(46,15 \%)$ & $28(43,75 \%)$ & $42(54,55 \%)$ \\
& Female & $7(53,85 \%)$ & $36(56,25 \%)$ & $34(44,15 \%)$ \\
Age of presentation $\geq 55$ & & $10(76,92 \%)$ & $50(78,12 \%)$ & $60(77.92 \%)$ \\
EPOC & & $1(7,69 \%)$ & $4(6,25 \%)$ & $5(6,49 \%)$ \\
Cardiopathy & & $2(15,38 \%)$ & $12(18,75 \%)$ & $4(18,18 \%)$ \\
Hypertension & & $7(53,85 \%)$ & $41(64,06 \%)$ & $15(62,3 \%)$ \\
Diabetes Mellitus & Yes & $1(7,69 \%)$ & $14(21,88 \%)$ & $12(15,48 \%)$ \\
Smoking & No & $0(0 \%)$ & $12(18,75 \%)$ & $19(63,63 \%)$ \\
& Former & $10(76.9 \%)$ & $39(60,93 \%)$ & $16(20,77 \%)$ \\
& $<25:$ & $3(23,07 \%)$ & $13(20,31 \%)$ & $16(20,77 \%)$ \\
BMI & $25-30$ & $2(15.38 \%)$ & $14(21.88 \%)$ & $34(44,15 \%)$ \\
& $30-35$ & $6(46,15 \%)$ & $28(43,75 \%)$ & $15(19,48 \%)$ \\
Hemoglobin & $>35:$ & $3(23,07 \%)$ & $12(18,75 \%)$ & $12(15,58 \%)$ \\
& $>10 \mathrm{~g} / \mathrm{dl}$ & $2(15.38 \%)$ & $10(15,62 \%)$ & $75(97,40 \%)$ \\
History of VTE & $<10 \mathrm{~g} / \mathrm{dl}$ & $13(16,9 \%)$ & $62(96,9 \%)$ & $2(2,60 \%)$ \\
Corticosteroids & & 0 & $2(3.1 \%)$ & $1(1,30 \%)$ \\
Chemotherapy (Temozolamide) & & 0 & $1(1,56 \%)$ & $74(96,10 \%)$ \\
Radiotherapy & & $13(100 \%)$ & $45(58,44 \%)$ \\
\hline
\end{tabular}

The distribution by sex of this population was $42(55.3 \%) /$ 34 (44.7\%), men and women, respectively. The median age in the moment of diagnosis was 66.42 years (range 38 to 85 years).

Regarding the functionality measured by the ECOG scale at the time of diagnosis, $42(55.3 \%)$ patients presented a performance status of 1 and 27 (35.5\%) 2; furthermore, 3 $(5.3 \%)$ registered a status of 3 , and only $2(2 \%)$ registered 0 .

In our series, $13(16.9 \%)$ of the 77 patients developed venous thromboembolism (VTE), of which 10 (61.54\%) presented deep venous thrombosis (DVT) and 3 (23.08\%) pulmonary thromboembolism (PE). The characteristics of the 
patients in each group are described in Table 1.

Exclusive stereotaxic biopsy was the procedure indicated in $13(16.9 \%)$ of the 77 patients. Partial or palliative resection was performed in $16(20.8 \%)$ of the 52 patients who had surgical intervention, and with apparent radical macroscopic removal in $36(46.8 \%)$ of the patients studied. Of all of them, $10(19.2 \%)$ presented VTE, this being a greater risk factor (OR 1,172).

The average time between radiological diagnosis and surgical intervention was 13.49 days (1-41, SD 7,76).

TMZ, is the most consolidated alternative within both complementary and palliative treatment of these patients, in our study, this medication was prescribed in $39(75 \%)$ of the 52 patients undergoing surgical intervention and in $5(38.5 \%)$ of the 13 in which only a biopsy was performed.

In our series, $10(22.2 \%)$ of the 44 patients who were treated with chemotherapy presented VTE (OR 1,407).

The average time of therapeutic delay after the surgical intervention and administration of chemotherapy was 58.68 days (21-231, SD 42,67) and those who had a biopsy 35.2 days (20-154 SD 14.67).

The onset of severe thrombocytopenia $(<50,000)$ as an adverse effect of toxicity was identified in $2(5 \%)$ of the 44

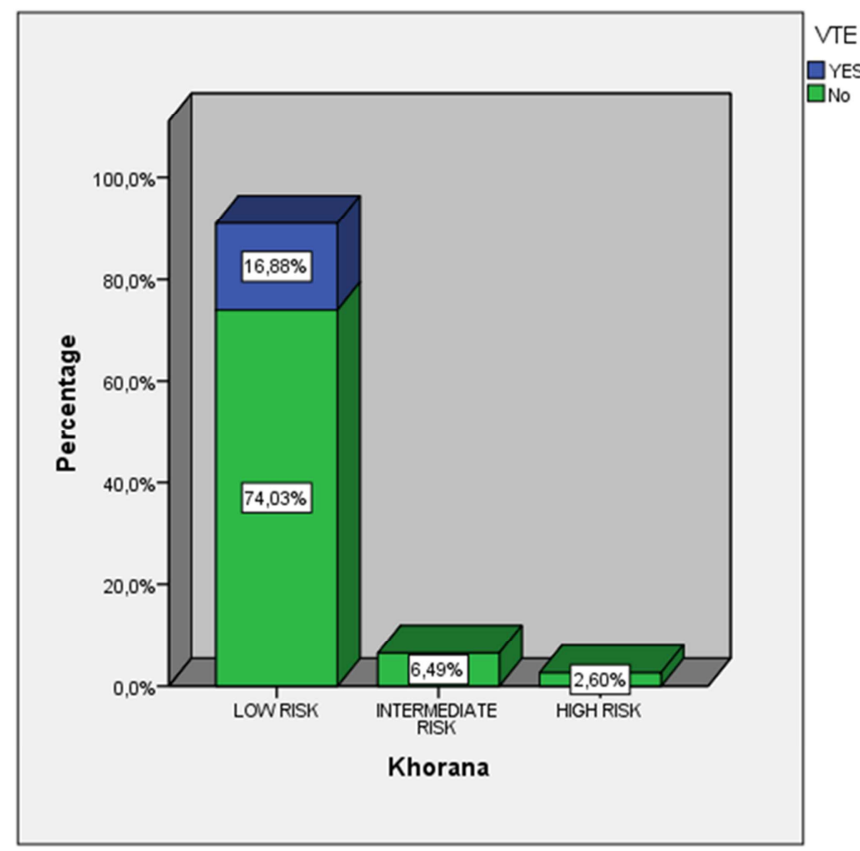

Figure 1. Comparison of thrombotic events after stratification of the predictor scales of Korana and the modified Asco 2013.

Considering the possibility of bleeding with the HASBLED scale, 74 patients (96.1\%) had a risk of less than 3. However, $2(2.6 \%)$ of the total 77 patients presented a major hemorrhagic event and 7 (7.9\%) Cerebral hemorrhage. All of them were at low risk of bleeding.

With a median 1 year follow-up, 64 (84.2\%) patients out of the 77 studied, died, with an average time after diagnosis cases who received TMZ, and moderate thrombocytopenia $(<100,000)$ in $3(7.5 \%)$ patients were detected.

With respect to hemorrhagic complications, $2(2.6 \%)$ of the patients presented a greater hemorrhagic event and 7 (7.9\%) cerebral hemorrhage, of which $4(44.4 \%)$ had prophylactic LMWHS and 5 (55.6\%) did not.

The administration of post-surgical external RT, with the dosage and volume of standard irradiation for this type of pathology was carried out in $40(77 \%)$ of the 52 patients subjected to surgical intervention and $4(31 \%)$ of the 13 that were subjected to diagnostic biopsy. $18.4 \%$ of all of them, developed VTE. Patients who were surgically intervened, had a greater risk of developing VTE (OR 1,172).

According to the predictive risk model for thrombosis in oncological patients Khorana, 37 (48.1\%) of the 77 patients studied, presented low risk of developing VTE, 38 (49.4\%) intermediate and $2(2.6 \%)$ high risk. (SE 0, 51). Of those who developed VTE, $5(38.5 \%)$ were in the low risk group and 8 $(61.5 \%)$ in the intermediate range. (Figure 1)

After stratification according to the modified ASCO 2013 scale, there was an intermediate risk of developing VTE 37 $(48.1 \%)$ and $40(51.9 \%)$ high risk, without finding any cases with low risk. (SE 0,5). (Figure 1)

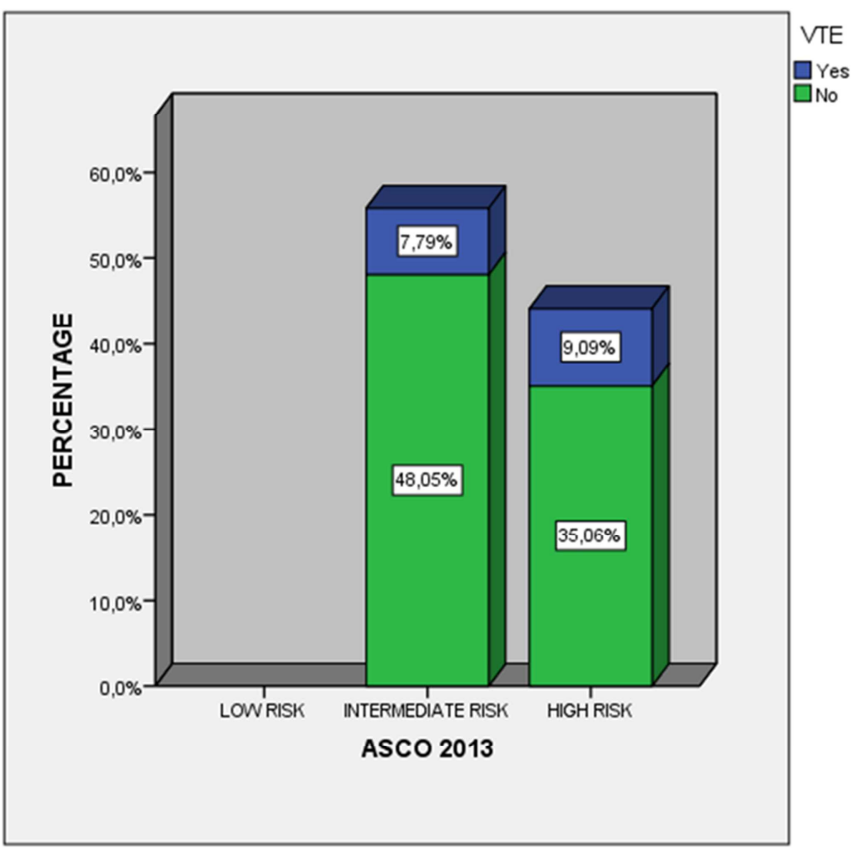

of 279.09 days (minimum 216.6, maximum 341.6 days) SE 31.8 .

As for the survival analysis of Kaplan Meyer, patients who received prophylactic treatment with LMWHs had a higher survival rate compared to those who did not receive it, with an average of 298.5 days compared to 239.3 respectively, with a $p>$ 0.05 in the Log Rank equation. SE 31.88 (Figure 2). 

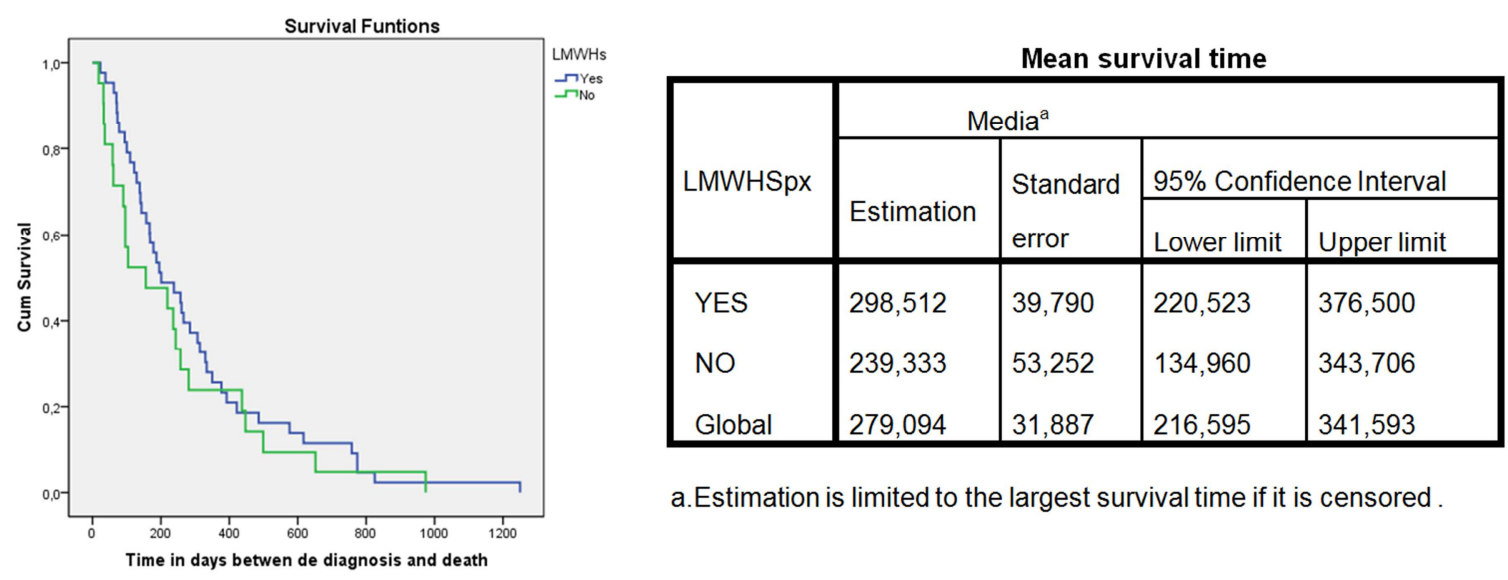

a. Estimation is limited to the largest survival time if it is censored

Figure 2. Survival comparison in patients who received LMWHS prophylactically.

A multiple linear regression model was applied to identify the risk factors associated with the thrombotic and haemorrhagic events. The variables shown in the table were included. By the method of backward methodical selection, the variables with a $\mathrm{P}>=0.15$ for the statistical result of Wald, the variable was eliminated, one by one, from the model, without finding any variable with a significant $p$ at the end.

\section{Discussion}

The GBM constitutes one of the solid tumors with a higher possibility of developing VTE, in addition to associated risk factors such as comorbidities, and surgical and pharmacological treatment.

In this retrospective review, a total of 77 patients diagnosed with GBM, at the General Hospital of Ciudad Real between 2012 and 2015 were studied; Its demographic and clinical features, laboratory variables, characteristics of the venous thromboembolic event, the variables related to the treatment of neoplasia and the treatment of thrombosis and complications of anticoagulation. The overall survival and the stratified overall survival according to the VTE, and to prophylaxis with low molecular weight heparin was also determined. Finally, it was identified how the Khorana scores were distributed compared to the ASCO score modified, in our patient population with thrombotic event.

In our study, $13(16.88 \%)$ patients developed VTE, similar to those that we can contrast in other publications; in a recent observational article it was estimated that the advocacy of VTE among patients with glioma was 16.1 events per 100 patients during the first six months post diagnosis [10]. In some series they even describe the odds of patients developing VTE being between 26 and 30\%, as described by Streiff et al in their multicenter prospective study of highgrade gliomas, in which $26 \%$ presented some thrombotic event [11].

Regarding the main epidemiological characteristics, (distribution by gender and average age) our series conforms to the main publications of the scientific literature. This indicated a predominance of this type of tumor in male patients.
The functionality or performance status measured through the ECOG scale at the time of diagnosis, showed that $90.8 \%$ of the patients in our study were between categories 1 and 2; $42(55.3 \%)$ and $27(35,5 \%)$ respectively, which means that mostly they were symptomatic patients which impacted their quality of life, with only $2.6 \%$ in category 0 . This data differs with the series published by Lacroix et al [12], in which they studied 416 patients with GBM, of which 233 were again diagnosed, $62 \%$ were in the ECOG equivalent between categories 1 and 2, being $15 \%$ in class 0 . With these results, it could be deduced that the patients of our study possibly attended a medical consultation with advanced symptoms.

When we analysed the clinical situation of patients at the time of the thrombotic event, we could identify that $75 \%$ of them were in categories 2 and 3; $5(41.7 \%)$ and $4(33.3 \%)$ respectively. Patients with a worse performance status would probably have less mobility and they would be less independent in their function, which would result in the stasis, a well-known risk factor for the development of thrombosis. There are various publications that include the Performance Status as a risk criterion for VTE in patients with solid tumors, among which is the evidence-based guide published by Shea et al [13] in a prospective study, $31 \%$ of patients with pulmonary cancer and poor functional status in chemotherapy, had a venous thromboembolic event compared to $15 \%$ of patients with good functionality. In spite of this, in the multicenter study carried out by Streif et al in 107 patients with high-grade gliomas, 26 had VTE, without having less ECOG compared to those who did not present such an event.

It is important to note that, within the group of 13 patients who presented VTE, 8 (53.84\%) had previous comorbidities, such as arterial hypertension which was the most predominant in $7(53.5 \%)$ of them, similar to the data displayed by Lim et al [14] with 57\% hypertensive patients, demonstrating in their study, to be at risk of thrombotic formation. In our series, the only data with a significant OR 1,02 is for COPD. Smoking is generally considered a risk factor for VTE, potentially mediated through conditions such as cancer; however, none of those who had VTE smoked, despite the fact that $23.07 \%$ were former smokers. 
The relative risk of suffering from malignant tumors in the obese population is increased (RR: 1.5 ), this being one of the categorization points in the Khorana classification [7]; which only takes into consideration patients with a BMI $>35 \mathrm{Kg}$ / $\mathrm{m}^{2}$, having a value of 1 . The prevalence of type III obesity in our series is $15.6 \%$ and of the patients who presented VTE it was $15.38 \%$, lower values than those of the Yust-Katz study [15], with $21.1 \%$ obesity $>$ II.

An important piece of data to highlight is that $100 \%$ of the patients who had thrombotic events were being medicated with corticosteroids (OR 1.049). This is consistent with previous studies that demonstrated that the use of corticosteroids generally increases the risk of thrombosis. Experimental studies have shown that the use of glucocorticoids increases the level of coagulation factors and fibrinogen, which subsequently increases the risk of VTE. Steroids are commonly prescribed to brain tumor patients with tumor progression and / or tumor edema. Therefore, steroid use likely reflects an increased tumor burden that may also increase the risk of VTE. In the study carried out by Yust-Katz et al [15], they highlighted that $76 \%$ of the patients diagnosed with GBM who had thrombotic events were treated with corticosteroids, similar results that we found according to the data published by Portillo et al. [16] in relation to patients with this type of tumor and thrombosis, of which $70 \%$ were also being treated with glucocorticoids.

$67.53 \%$ of the population studied underwent some type of surgical intervention (biopsy, partial or total resection), of which 19.2\% had some thrombotic event (OR 1.172). The literature has highly variable data in this regard, on the one hand we find the study by Kaewborisutsakul et al. [17], in which they describe the incidence of thrombotic events after craniotomies in patients with brain tumors, of which only $10.2 \%$ of 177 cases studied had such an event, however in this study they describe all types of tumors and only $14.7 \%$ were GBM and they do not indicate in which type of tumor VTE was more frequent. In contrast to this, in the RIETE registry study, $36 \%$ of patients with VTE had recent surgery [16].

$76.92 \%$ of the patients who presented a thrombotic event received chemotherapy with TZM (OR 1.407). This figure is consistent with the series of patients reviewed in which the use of chemotherapy ranges from $42.4 \%$ to $77 \%$ [18]. In the YustKatz series, $81 \%$ of the patients received chemotherapy (15); In contrast, in the one described in the RIETE study, $49 \%$ received it [16].

There are models aimed at identifying subgroups of patients with a high risk of VTE, being able to be useful to assess prophylaxis with LMWHS, with the most used Khorana model, since it was validated in more than 10,000 oncological patients [7].

The present study showed that $74.03 \%$ of patients who developed thrombosis had a low-risk Khorana score (less than three). That is, this model was not a predictor of thrombosis in most of our patients, making us suspect a very low positive predictive value. The original study of Khorana
[7] showed rates of thromboembolic disease in $3.6 \%$ derivation and validation cohorts in the low risk group and $13.8 \%$ in the high-risk group; coinciding with the low positive predictive value of the current study; also taking into account that most patients in that investigation had a good functional condition, and that there was no adequate representation of tumors of high thrombogenic potential such as those that affect the brain [19], that causes doubt about its clinical usefulness in this type of neoplasms. Thus, the scale modified by ASCO 2013 [20], in our series showed more real data, of which $46.2 \%$ of patients with thrombosis had an intermediate risk of developing it and $53.8 \%$ high risk.

Low molecular weight heparins were the most used drugs, with enoxaparin being most used in $49(63.6 \%)$ patients in a prophylactic manner and as long-term treatment after thrombotic event in $5(55.56 \%)$ of them. This reflects a high adherence of professionals to the thrombosis management guidelines associated to cancer of the ACCP, NCCN [20, 21]. The data for heparin use are even higher than those reported in European observational studies such as Den Extern et al, in which the use of heparins versus warfarinants before and after 2008 were compared, finding 381 patients with thrombosis associated with cancer, heparin was used in $23 \%$ of cases before 2008 compared to $67 \%$ after [22]. In any case, in the RIETE data [15], the percentage of patients with prophylactic treatment with LMWHS reached $93 \%$ and long-term at $78 \%$.

A dreaded complication of the use of heparin concomitantly with chemotherapy is thrombopenia, in our series, of the 40 patients who received chemotherapy, we found in $4(10 \%)$ of them severe thrombocytopenia with a value less than $50,000 / \mathrm{mcl}$, within this group 3 of them also had treatment with LMWHS at prophylactic doses. This data is less than what was expected in other studies, as in Gerber et al, who refer thrombocytopenia grade 3 and $4(<50,000 /$ MCL) in $10(19 \%, 95 \%$ CI, 10\% -33\%) in a descriptive study of 52 patients with GBM and concomitant chemotherapy with TMZ [23].

The results of CLOT included an impressive reduction in the recurring VTE of $17 \%$ to $9 \%$ that favoured the arm of LMWHS, without a difference in major haemorrhage $(p=0.002)$ [24]. This result and similar ones support the use of LMWHS as a preferred initial therapy for patients with malignant glioma. LMWHS is associated with other clinical advantages, compared to oral anticoagulants, including the absence of monitoring in the laboratory and minimum interaction between medications and food.

We evaluated the risk of bleeding through the HASBLED scale, resulting in $3.9 \%$ with high risk. As for haemorrhagic complications, only $2.6 \%$ of patients presented a greater haemorrhagic event and $7.9 \%$ cerebral haemorrhage, of which only $2.6 \%$ were in the group that developed a thrombotic event and had treatment with LMWHS, which agrees with other studies, due to high-grade gliomas, the risk reported from spontaneous haemorrhage is between 2 and $8 \%$, with higher GBM rates and anaplastic oligodendroglioma [25, 26]. This seems to indicate that this scale does not represent a good predictor of the risk of bleeding, since it was designed 
to evaluate the risk of major bleeding in anticoagulated patients by atrial fibrillation. In the study by Yust-Katz et al, $4.7 \%$ of patients presented cerebral haemorrhage and with similar data, of greater haemorrhage [15].

Thus, we can infer that the risk of bleeding in patients affected by glioblastoma is evidently low, which agrees with the current literature. In fact, according to the study of Cote et al., 2014 neurosurgical patients who developed VTE, presented greater risk of dying by pulmonary thromboembolism than by major bleeding despite anticoagulation, with $2.9 \%$ of cases of intracranial haemorrhage in those with cerebral neoplasm [27].

After a 1-year follow-up, 64 patients $(84.2 \%)$ of the 73 studied, died, with an average of 39.46 days from the diagnosis. According to the literature, only $5 \%$ of the patients with a diagnosis of GMB survive 5 years. 14 months is the average life expectancy that can be offered after full tumor resection, as well as radio and chemotherapy coadjuvant [28].

Despite it has not been demonstrated in our study that the presence of thrombotic event is a risk factor that accelerates the end of the life of these patients, it is interesting to note that those who had prophylactic treatment with LMWHS had a higher survival rate, probably related to the theory of the empalized, in which they were inhibited neoangiogenesis and thus tumor growth (6), In the study of Zincircioglu et al [29] carried out in 30 patients diagnosed with GBM, in which 17 patients received LMWHS at prophylactic doses concomitantly to chemo and radiotherapy, with a global survival of 69 and 44 weeks $(\mathrm{p}=0.095), 1$ year survival 84.6 and $41.2 \% \quad(\mathrm{p}=0.016)$ and survival at 2 years 38.5 and $5.9 \%$ in LMWHS + and LMWHS-, respectively $(p=0.061)$; Even though the number of cases was small, a greater survival rate can be clearly appreciated in the patients who received the LMWHS.

\section{Conclusions}

The demographic and clinical characteristics of the patients analysed with thrombosis associated with GBM are similar to those reported in international investigations. The frequency and type of major bleeding are like those reported in records from other latitudes.

Unfortunately, the predictive scale of Khorana used for the risk analysis of VTE in other types of cancer was not validated in our study of patients with GBM and a specific alternative scale for this patient population has not been developed. The modified ASCO scale is the one which is closest to our results.

The creation of a precise predictive model would allow a randomized clinical trial focused on preventive VTE measures in patients with high-risk GBM and would help to delineate the benefits of the use of prophylactic anticoagulation.

Long-term prophylaxis with LMWHS has demonstrated a reduction of thrombotic events without significantly increasing the fatal haemorrhagic events, also demonstrating greater independent survival of the VTE. Randomized prospective studies are needed to demonstrate their benefit.

\section{Limitations}

Being a retrospective study, a percentage of the data may not be measured accurately if it was not documented in the reports according to the scales we used.

Despite the LMWHS demonstrated a greater survival rate, we cannot recommend the universal use of this medication in patients with GBM without having a prospective randomised study.

\section{References}

[1] Ostrom QT, Gittleman H, XI J, Kromer C, Wolinsky Y, Krinchko C, et al. CBTRUS Statistical Report: Primary Brain and Central Nervous System Tumors Diagnosed in the United States in 2009-2013. Neuro Oncology. 2016; 18 (suppl 5): p. v1-v75.

[2] Gussoni G, Frasson S, La Regina M, Di Micco P, Monreal M, Investigators. R. Three-month mortality rate and clinical predictors in patients with venous thromboembolism and cancer. Findings from the RIETE registry. Thrombosis research. 2013 January; 131 (1): p. 24-30.

[3] Lyman G, Khorana A, Falanga A, Clarke-Pearson D, Flowers C, Jahanzeb M, et al. American Society of Clinical Oncology guideline: recommendations for venous thromboembolism prophylaxis and treatment in patients with cancer. Journal of Clinical Oncology. 2007 De ember; 25 (34): p. 5490-505.

[4] Versteeg H, Schaffner F, Kerver M, Petersen H, Ahamed J, Felding-Habermann B, et al. Inhibition of tissue factor signaling suppresses tumor growth. Blood. 2008 January; 111 (1): p. 190-9.

[5] Ludwig R, Alban S, Boehncke W. Structural requirements of heparin and related molecules to exert a multitude of antiinflammatory activities. Mini Reviews in Medicinal Chemistry. 2006 September; 6 (9): p. 1009-23.

[6] Martínez-González A, Calvo G, Pérez Romasanta L, PérezGarcía V. Hypoxic cell waves around necrotic cores in glioblastoma: a biomathematical model and its therapeutic implications. Bulletin of Mathematical Biology. 2012 December; 74 (12): p. 2875-96.

[7] Khorana A, Kuderer N, Culakova E, Lyman G, Francis C. Development and validation of a predictive model for chemotherapy-associated thrombosis. Blood. 2008 May; 111 (10): p. 4902-7.

[8] Lyman G, Khorana A, Kuderer N, Lee A, Arcelus J, Balaban E, et al. Venous thromboembolism prophylaxis and treatment in patients with cancer: American Society of Clinical Oncology clinical practice guideline update. Journal of Clinical Oncology. 2013 June; 31 (17): p. 2189-204.

[9] Sideras K, Schaefer P, Okuno S, Sloan J, Kutteh L, Fitch T, et al. Low-molecular-weight heparin in patients with advanced cancer: a phase 3 clinical trial. Mayo Clinic Proceedings. 2006 June; 81 (6): p. 758-67.

[10] Semrad T, O'Donnell R, Wun T, Chew HHD, Zhou H, White R. Epidemiology of venous thromboembolism in 9489 patients with malignant glioma. Journal of Neurosurgery. 2007 April; 106 (4): p. 601-8. 
[11] Streiff M, Ye X, Kickler T, Desideri S, Jani J, Fisher J, et al. A prospective multicenter study of venous thromboembolism in patients with newly-diagnosed high-grade glioma: hazard rate and risk factors. Journal of neuro-oncology. 2015 June; 142 (2): p. 299-305.

[12] Lacroix M, Abi-Said D, Fourney D, Gokaslan Z, Shi W, DeMonte F, et al. A Multivariate Analysis of 416 Patients With Glioblastoma Multiforme: Prognosis, Extent of Resection, and Survival. Journal of Neurosurgery. 2001 August; 95 (2): p. 190-198.

[13] Shea-Budgell M, Wu C, Easaw J. Evidence-based guidance on venous thromboembolism in patients with solid tumours. Current Oncology. 2014 June; 21 (3): p. e504-e514.

[14] Lim G, Ho C, Roldan-Urgoti G, Leugner D, Easaw J. Risk of Venous Thromboembolism in Glioblastoma Patients. Cureus. 2018 May; 10 (5): p. e2678.

[15] Yust-Katz S, Mandel J, Wu J, Yuan Y, Webre C, Pawar T, et al. Venous thromboembolism (VTE) and glioblastoma. Journal of Neuro-Oncology. 2015 August; 124 (1): p. 87-94.

[16] Portillo J, de la Rocha I, Font L, Braester A, Madridano O, Díaz Peromingo J, et al. Venous thromboembolism in patients with glioblastoma multiforme. Thrombosis Research. 2015 December; 136 (6): p. 1199-203.

[17] Kaewborisutsakul A, Tunthanathip T, Yuwakosol P, Inkate S, Pattharachayakul S. Incidence and Risk Factors for Venous Thromboembolism Following Craniotomy for Intracranial Tumors: A Cohort Study. Asian Journal of Neurosurgery. 2020 January-March; 15 (1): p. 31-38.

[18] Dolovich L, Ginsberg J, Douketis J, Holbrook A, Cheah G. A meta-analysis comparing low-molecular-weight heparins with unfractionated heparin in the treatment of venous thromboembolism: examining some unanswered questions regarding location of treatment, product type, and dosing frequency. Archives of Internal Medicine. 2000 January; 160 (2): p. 181-8.

[19] Thaler J, Ay C, Pabinger I. Venous thromboembolism in cancer patients -Risk scores and recent randomised controlled trials. Thrombosis and Haemostasis. 2012 December; 108 (6): p. $1042-8$.

[20] Lyman G, Bohlke K, Khorana A, Kuderer N, Lee A, Arcelus J, et al. Venous thromboembolism prophylaxis and treatment in patients with cancer: american society of clinical oncology clinical practice guideline update 2014. Journal of Clinical Oncology. 2015 february; 33 (6): p. 654-6.
[21] Streiff M, Holmstrom B, Ashrani A, Bockenstedt P, Chesney C, Eby C, et al. Cancer-Associated Venous Thromboembolic Disease, Version 1.2015. Journal of the National Comprehensive Cancer Network. 2015 September; 13 (9): p. 1079-95.

[22] den Exter P, Hooijer J, can der Hulle T, Van Oosten J, Dekkers O, Klok F, et al. Vitamin K Antagonists Compared to LowMolecular-Weight Heparins for Treatment of CancerAssociated Venous Thromboembolism: An Observational Study in Routine Clinical Practice. An Observational Study in Routine Clinical Practice. Thrombosis and Haemostasis. 2017 November; 117 (11): p. 2163-2167.

[23] Gerber D, Grossman S, Zeltzman M, Parisi M, Kleinberg L. The impact of thrombocytopenia from temozolomide and radiation in newly diagnosed adults with high-grade gliomas. Neuro-Oncology. 2007 January; 9 (1): p. 47-52.

[24] Lee A, Levine M, Baker R, Bowden C, Kakkar A, Prins M, et al. Low-molecular-weight heparin versus a coumarin for the prevention of recurrent venous thromboembolism in patients with cancer. The New England Journal of Medicine. 2003 July; 349 (2): p. 146-53.

[25] Bitoh S, Hasegawa H, Ohtsuki H, Obashi J, Fujiwara M, Sakurai M. Cerebral neoplasms initially presenting with massive intracerebral hemorrhage. Surgical Neurology. 1984 July; 22 (1): p. 57-62.

[26] Lieu A, Hwang S, Howng S, Chai C. Brain tumors with hemorrhage. Journal of the Formosoan Medical Association. 1999 May; 98 (5): p. 365-7.

[27] Cote L, Greenberg S, Caprini J, Stone J, Arcelus J, LópezJiménez L, et al. Outcomes in neurosurgical patients who develop venous thromboembolism: a review of the RIETE registry. Clinical and Applied Thrombosis/Hemostasis. 2014 November; 20 (8): p. 772-778.

[28] Delgado-López P, Corrales-García E. Survival in Glioblastoma: A Review on the Impact of Treatment Modalities. Clinical \& translational oncology. 2016 November 1062-1071; 18 (11).

[29] Zincircioglu S, Kaplan M, Isikdogan A, Cil T, Karadayi B, Dirier A, et al. Contribution of low-molecular weight heparin addition to concomitant chemoradiotherapy in the treatment of glioblastoma multiforme. Jpurnal of the Balkan Union Oncology. 2012 January-March; 17 (1): p. 124-7. 